\title{
Editorial
}

\section{Green Energy and Sustainability journal-research supporting global energy diversity for a healthier planet}

George Papadakis

Received: 19 Apr 2021

Accepted: 19 Apr 2021

Published: 5 Jul 2021

Copyright: (c) 2021 by the author(s). This is an Open Access article distributed under the terms of the Creative Commons License Attribution 4.0 International (CC BY 4.0), which permits unrestricted use, distribution, and reproduction in any medium or format, provided the original work is correctly credited.

Publisher's Note: Pivot Science Publication Corp. remains neutral with regard to jurisdictional claims in published maps and institutional affiliations.
Department of Natural Resources and Agricultural Engineering, Agricultural University of Athens (AUA), Iera Odos 75, Athens 11855, Greece; E-Mail: gpap@aua.gr.

Common sense suggests that there are sufficient forums that publish scientific research on energy efficiency and sustainability and renewable energy sources, so the question arises: "Why start another journal for publications on green energy research?" Our current modes of energy production have had adverse impacts on the environment, and we cannot continue harming the planet we live on to meet our ever-increasing energy needs. Open access dissemination of information on energy technologies and the interaction between energy production and the environment is vital to the development of a global knowledge base. The Green Energy and Sustainability journal (GES) is a celebration of the diversity of green energy sources and technologies and the constant new discoveries on the close connection between energy production, the environment, and ecosystems in general.

Burning biomass has been the source of energy for heating, cooking, and lighting for thousands of years. From the late 1800s, fossil fuels (coal, oil, and natural gas) and hydroelectricity were the main sources of energy; but since the 1990s, the volume and share of total global energy consumption from renewable energy sources, solar, wind, biofuels, and geothermal have increased significantly.

It has been demonstrated and confirmed that adoption of green energy practices is highly effective in mitigating climate change. Primary dependence on fossil fuels and inefficient and outdated coal-fired power stations are some of the main reasons for the steadily increasing level of global greenhouse gas emissions. This has raised the average global temperature and seriously affected air quality and human health. The positive role of renewable energy sources has been recognized internationally by the Paris Agreement on Climate Change as well as the Sustainable Development Goals.

Research on renewable energy and energy efficiency has grown rapidly over the last three decades and continues to expand at an impressive rate. The industry is also evolving rapidly and creating 
opportunities for future up-scaling to achieve a real, global energy transformation. These opportunities are related to the following: Many parts of the world are still experiencing energy shortages and are eager for alternatives to fossil fuels to secure energy production and supply; the role of renewable energy in mitigating climate change has been recognized as a proven means to meet national climate targets in line with the Paris Agreement; the dramatic reduction in cost of renewable energy production has placed such alternatives in reach of developing countries; there is a growing interest on the part of energy entrepreneurs to expand into global markets; and, the increasing support of international organizations, governments and the global community are creating a favorable environment for renewable energy investment.

The vision of the GES journal is to create an open access channel for research results supporting the global energy community. Publication topics will reflect a variety of subjects ensuring highly useful and relevant content for both stakeholders and the general public. Special issues will be set up on specific topics, as recommended by interested authors and editors. The greatest integrity of the peer review process and ethics in publishing will be maintained throughout.

All contributing authors will receive the careful attention and assistance of the editorial staff in reviewing submitted papers, which will definitely contribute to the acceptance and recognition of GES by the scientific community.

The quality of papers accepted for publication in GES is safeguarded by a dedicated Editorial Board of 20 prominent scholars. Our collective goal is for GES to be globally recognized as the go-to publication for innovative, high-quality research on a wide range of topics in the diverse and broad fields of energy and sustainability. This goal depends on authors with a strong commitment to their work who dare to come up with radical ideas and who want to encourage discussion and further research through a high-profile, globally accessible publication. We invite you to submit original research papers, reviews and short communications for peer review and prospective publication in GES, as well as to send us your ideas for future issues focusing on hot topics in the field of green energy and sustainability. Your submissions are vital for harnessing the potential of GES as a source for contributing significantly to the global scientific knowledge base, and ultimately to meeting our planet's energy needs sustainably.

Cite this article: Papadakis G. Green Energy and Sustainability journal-research supporting global energy diversity for a healthier planet. Green Energy Sustain 2021; 1(1):0001. https://doi.org/10.47248/HKOD902101010001. 